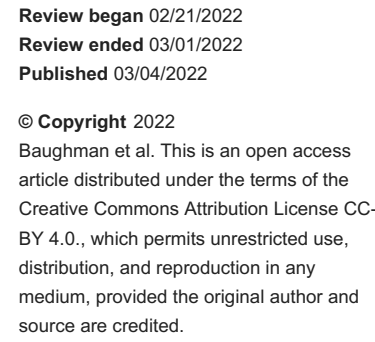

\section{Racial Disparity in Pediatric Radiography for Forearm Fractures}

\author{
Derek J. Baughman ${ }^{1}$, Taofeek Akinpelu ${ }^{1}$, Abdul Waheed ${ }^{2,3}$, Thomas Trojian ${ }^{4}$ \\ 1. Family Medicine, WellSpan Good Samaritan Hospital, Lebanon, USA 2. Family Medicine, Wellspan Good Samaritan \\ Hospital, Lebanon, USA 3. Family and Community Medicine, Penn State University College of Medicine, Milton S. \\ Hershey Medical Center, Hershey, USA 4. Sports Medicine, WellSpan York Hospital, York, USA
}

Corresponding author: Derek J. Baughman, baughman.derek@gmail.com

\begin{abstract}
Introduction: The most common pediatric fractures involve the upper extremity. But there is limited study on racial disparity in diagnostic radiography for pediatric fractures. The literature has described the diagnostic accuracy of alternative diagnostic modalities with promising evidence of its ability to mitigate health inequity in primary care. Our objective was to understand if racial disparity exists in radiography for pediatric fractures.
\end{abstract}

Methods: In this four-year retrospective cohort study, we analyzed rates of radiographic imaging and abnormal radiograph detection in 4280 pediatric patients (ages 3-18 years) who presented with chief complaints of arm or wrist pain and trauma-related International Classification of Diseases 10th Revision (ICD-10) codes. We compared White children to all other races and stratified by emergency departments (ED) vs all other primary care ambulatory service lines.

Results: Non-White patients had lower imaging rate differences and lower odds receiving imaging in both ambulatory settings $(0.65915, \mathrm{P}=0.0162 ;-5.4 \%, \mathrm{P}=0.0143)$ and in EDs $(0.7732, \mathrm{P}=0.0369 ;-4.7 \%, \mathrm{P}=$ $0.0368)$. Additionally, non-Whites in the ED had lower rates and lower odds of abnormal radiographs $(-7.3 \%$, $\mathrm{P}=0.0084 ; 0.6794, \mathrm{P}=0.0089)$.

Conclusion: Non-White patients seen in emergency and ambulatory settings had lower imaging rates for traumatic arm and wrist pain compared to White patients, indicating a healthcare disparity in pediatric imaging. Higher-level studies investigating the effect of social determinants of health, more detailed patient data, and provider bias on facture care equity are needed to understand underlying reasons for observed differences.

Categories: Family/General Practice, Pediatrics, Orthopedics

Keywords: healthy equity, population health management, pediatric fractures, pediatrics, healthcare disparities

\section{Introduction}

Racial disparities in medicine are well-known. Non-White minorities make up a significant portion of the United States (US) population; they have decreased access to care and increased spending, especially in emergency and inpatient care [1-4]. These disparities also exist in pediatric populations. It is well described in the literature that pediatric insurance status impacts the amount of primary care received [5]. Compared with Whites, racial minorities have lower levels of health insurance coverage [6] and higher levels of costsharing, preventing low-income, highest-need populations from receiving needed care [7]. In addition, costsharing (i.e., higher out-of-pocket costs) is associated with worse treatment compliance and higher hospitalization rates [8] - a feed-forward mechanism that inflates cost burden.

Efforts to mitigate costs and increase accessibility have emerged because of the disproportionate use of emergency services by racial minorities [1]. Arm fractures are among the top reasons that pediatric populations use the emergency room/emergency department (ED) [8]. Forearm fractures in pediatric populations account for up to $50 \%$ of all fractures seen in children [9]. The known disadvantages to traditional radiography include higher cost burden, longer lengths of stay, impaired patient experience, and unnecessary ionizing radiation exposure. Accordingly, alternative diagnostic modalities to traditional radiography have gained recognition in the literature. Current evidence suggests that point-of-care ultrasound (POCUS) has comparable accuracy as radiography in diagnosing upper extremity fractures in children [9].

The advantages of POCUS include avoiding ionizing radiation, enhancing patient accessibility, and decreasing length of stay [9]. Moreover, POCUS can visualize indirect signs of fracture (hematoma, periosteum detachment) and evaluate soft tissue damage such as muscle edema and tendinopathy [10]. Specifically, POCUS can improve patient comfort in children, expedite diagnosis, and decrease cost without jeopardizing care [9]. In the hands of experienced providers, POCUS in primary care can detect pediatric 
Although the literature has described access and cost barriers for pediatric minorities, there is limited evidence regarding racial discordance in pediatric forearm care. Additionally, little is known about the diagnostic accuracy of pediatric fractures within racial minorities - a critical step to understanding if the need exists for improved access to imaging. Thus, our study aim was to discover if there is racial disparity in the access to imaging or the detection rate of pediatric forearm fractures in an expanded health system.

\section{Materials And Methods}

We retrospectively examined the imaging and abnormal radiograph rates of upper extremity fractures in pediatric patients in the age group of 3-18 years. SlicerDicer is a self-service cohort query tool in EPIC (Epic Systems Corporation, Verona, Wisconsin) that allows users to analyze patient population-level data. A patient data model was used in SlicerDicer to mine patient visits throughout the health system from April 1, 2017 , to July 31, 2021. Only the emergency services and pertinent ambulatory service lines were selected for inclusion. We measured if patients received imaging (x-ray, CT, or MRI), and if a subsequent fracture diagnosis code was added within three days of this imaging. In addition, we compared imaging rates obtained in those patients that self-identified as White and patients that did not self-identify as White, then stratified this comparison by venue (ED or Ambulatory).

The WellSpan Health Human's Protections Office deemed this study exempt from full review (1804211-1). The Strengthening the Reporting of Observational Studies in Epidemiology (STROBE) format for cohort studies was followed.

The data search session in EPIC was built according to the schema represented in Figure 1. To ensure that we accurately captured etiologies that warrant imaging for forearm fractures, ICD-10 code groupers for fall (ICD-10-CM W19*) and trauma (including V, Z, and T code categories) were added. Thus, the search comprehensively linked trauma-related codes to the chief complaint, facilitating the exclusion of nontraumatic etiologies that would have otherwise falsely inflated the denominator (for example, encounters for hand pain or superficial lacerations). 


\section{Cureus}

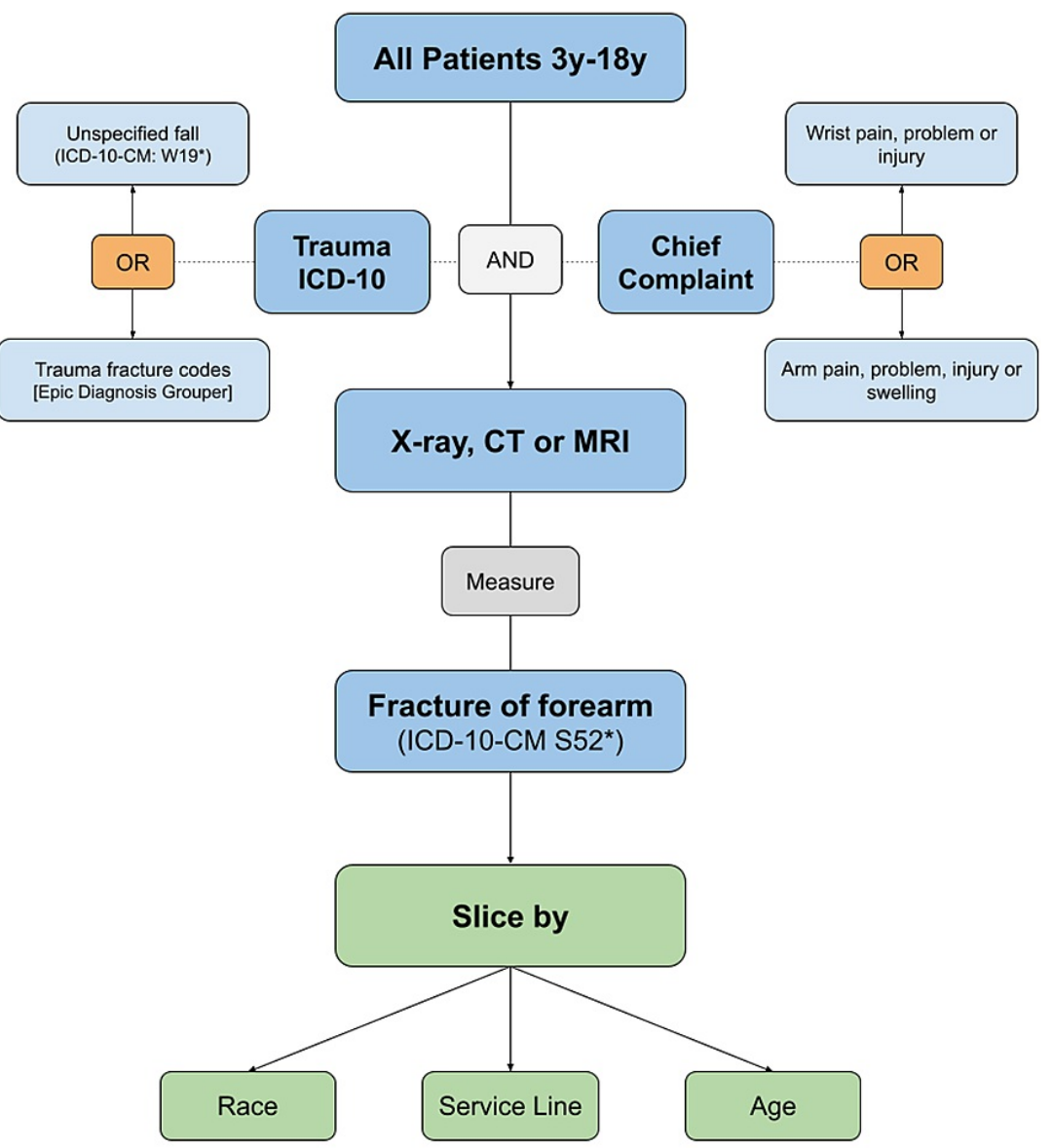

\section{FIGURE 1: SlicerDicer patient data model schema with filter prioritization}

ICD-10: International Classification of Diseases 10th Revision; ICD-10CM S52*: ICD-10 Diagnosis Code S52 (Fracture of forearm); ICD-10-CM: W19*: ICD-10 Diagnosis Code W19 (Unspecified fall)

To calculate the abnormal imaging rate, in the denominator, we included all patients with chief complaints and traumatic ICD-10 codes. In the numerator, we measured all patients who had radiographic imaging completed at the same encounter and who were diagnosed with a forearm fracture (EPIC groupers for forearm fractures were used to capture ICD-10-CM S52* diagnostic codes in SlicerDicer). To avoid redundantly counting patients who may have had multiple radiographic images within the four-year timeframe, we linked fracture diagnosis measurement to a three-day timeframe. This method ensured that the image counted in the numerator was unique to the patient encounter where the image was ordered. In addition, the three-day window was designated to accommodate reflection of "read" status in the electronic medical records (EMR); this avoided underrepresenting the numerator during the brief time when imaging was completed but not officially documented by radiologists.

The conglomerate term "non-White" was used to include a representation of racial categories in the sample: Asian, Black, or African American, American Indian or Alaska Native, Native Hawaiian or Other Pacific Islander, "unknown," and "other." This was due to very limited numbers of racial categories for adequate sub-comparisons (for example, the "unknown" category contained only 13 patients).

We identified care venue as a potential bias in our study given the inherent nature of the ED and lower threshold for obtaining imaging. However, we controlled this by stratifying our data by care venue emergency service lines vs. all other ambulatory primary care service lines.

\section{Statistical analysis}

We used gross numbers of patient encounter data obtained from SlicerDicer within Microsoft Excel (Microsoft Corp., Redmond, Washington) to calculate both rates of imaging and diagnosis and rate differences between sub-groups. To obtain subgroups, we "sliced" the results for patients according to age, race, and care venue (Figure 1). Then, Medclac's "N-1" Chi-squared calculator 


\section{Cureus}

(MedCalc Software Ltd, Oostende, Belgium) [12] was used to determine statistical significance $(\mathrm{P}<0.05)$. In Chi-squared tests, we compared White patients to all other races.

\section{Results}

Table 1 reveals the demographic data of the pediatric population. Patients presenting for traumatic arm and wrist pain were predominantly White $(80.1 \%)$ with the highest representation in the age group of 14-18 years (41.5\%), followed by ages $10-14$ years (32.1\%) and ages $<10$ years (26.4\%). Most of the encounters were in emergency settings (79.4\%).

\begin{tabular}{|c|c|c|c|c|c|}
\hline & Total with traumatic arm/wrist pain & Total patents imaged & Total with diagnosed fracture & Imaging rate * & Abnormal radiograph rate \pm \\
\hline Total patients $\ddagger$ & 4280 & 1914 & 504 & $44.7 \%$ & $26.3 \%$ \\
\hline White patients & 3485 & 1539 & 425 & 44.2\% & $27.6 \%$ \\
\hline Non-White patients & 795 & 375 & 79 & $47.2 \%$ & $21.1 \%$ \\
\hline Age total & 4280 & 1904 & 505 & 44.5\% & $26.5 \%$ \\
\hline Age $<10$ years & 1159 & 502 & 181 & $43.3 \%$ & $36.1 \%$ \\
\hline Age 10-14 yrars & 1333 & 611 & 184 & $45.8 \%$ & $30.1 \%$ \\
\hline Age $14-18$ years & 1788 & 791 & 140 & $44.2 \%$ & $17.7 \%$ \\
\hline Service line totals & 4280 & 1914 & 504 & $44.7 \%$ & $26.3 \%$ \\
\hline Emergency services & 1972 & 1520 & 416 & 77.1\% & $27.4 \%$ \\
\hline White patients & 1520 & 1188 & 344 & $78.2 \%$ & $29.0 \%$ \\
\hline Non-White patients & 452 & 332 & 72 & $73.5 \%$ & $21.7 \%$ \\
\hline Non-emergency services total & 2308 & 394 & 88 & $17.1 \%$ & $22.3 \%$ \\
\hline White patients & 1965 & 351 & 81 & $17.9 \%$ & $23.1 \%$ \\
\hline Non-White patients & 343 & 43 & 7 & $12.5 \%$ & $16.3 \%$ \\
\hline Payer type totals & 4280 & 1914 & 504 & $56.26 \%$ & $26.79 \%$ \\
\hline Commercial (Highmark, WellSpan, Blue Cross + other smaller payers) & 2,392 & 1,062 & 298 & $44.38 \%$ & $28.10 \%$ \\
\hline Medicaid \& Government & 1,723 & 784 & 190 & $45.52 \%$ & $24.25 \%$ \\
\hline Self Pay/Other & 165 & 68 & 16 & $41.39 \%$ & $22.79 \%$ \\
\hline
\end{tabular}

TABLE 1: Summary of pediatric patient access and diagnostic accuracy of imaging for chief complaints of arm or wrist "pain," "injury," or "problem" from April 1, 2017, to July 31, 2021

* Defined as (total number of patients imaged/total of patients with CC), serves as a proxy to understand the impact of care access.

\pm Defined as (total number of patients diagnosed with a forearm fracture/total number of patients imaged), serves as a proxy to understand impact of unnecessary (wasteful) imaging.

$\ddagger$ Total pediatric patients were arrived at by tabulating race counts as this characteristic did not change over the timeframe. Note that total age group numbers sum to the matching total by race.

In comparing service lines, there were significantly higher rates in the ED compared to ambulatory service lines for both imaging rates (ED: $77.1 \%$, Ambulatory: 17.1, difference: $60.0 \%$; CI: $57.5175 \%$ to $62.3316 \%$, Chisquared: $1548.047, \mathrm{P}<0.0001$ ) and abnormal radiograph rates (ED: $27.4 \%$, Ambulatory: $22.3 \%$, difference: 5.1 $\% ; 0.2182 \%$ to $9.5674 \%$, Chi-squared: $4.191, \mathrm{P}=0.0406$ ). In combining service lines (ED + ambulatory), there were insignificant differences between Whites versus non-Whites for imaging rates (ED: $44.2 \%$, Ambulatory: 42.7\%, difference: $3.01 \%$; CI: $-0.8144 \%$ to $6.8534 \%$, Chi-squared: $2.372, \mathrm{P}=0.1235$ ) and but there were significant differences for abnormal radiograph rates (ED: $26.7 \%$, Ambulatory: $21.1 \%$, difference: $6.5 \%$; CI: $1.5807 \%$ to $10.9575 \%$, Chi-squared: $6.565, \mathrm{P}=0.0104$ ). 
When stratified by care venue, non-Whites consistently had lower imaging rates and lower abnormal radiograph rates (Table 2). Imaging rate differences were statistically significant in the $\mathrm{ED}$ (White: $78.2 \%$, non-White: $73.5 \%$; difference: $4.7 \%, \mathrm{P}=0.0368$ ) and in ambulatory service lines (White: $17.9 \%$, non-White: $12.5 \%$; difference: $5.4 \%, \mathrm{P}=0.0143$ ). Abnormal radiograph rate differences were statistically significant in the ED (White: $29.0 \%$, non-White: $21.7 \%$; difference: $7.3 \%, \mathrm{P}=0.0084$ ) but not in the ambulatory service line (White:23.1\%, non-White: $16.3 \%$; difference: $6.8 \%, \mathrm{P}=0.3130$ ).

\begin{tabular}{|c|c|c|c|c|c|c|}
\hline & & White & Non-White & Chi sq $\Delta$ & Confidence interval & P-value \\
\hline \multirow{2}{*}{ Emergency Services Only } & Imaging rate ** & $78.20 \%$ & $73.50 \%$ & $4.7 \%$ Chi-squared: 4.360 & $(0.2854 \%$ to $9.4023 \%)$ & 0.0368 \\
\hline & Abnormal radiograph rate \pm & $29.00 \%$ & $21.70 \%$ & $7.3 \%$ Chi-squared: 6.946 & $(1.9332 \%$ to $12.1762 \%)$ & 0.0084 \\
\hline \multirow{2}{*}{ Ambulatory Services Only } & Imaging rate * & $17.90 \%$ & $12.50 \%$ & $5.4 \%$ Chi-squared: 6.005 & (1.1534\% to $8.9545 \%)$ & 0.0143 \\
\hline & Abnormal radiograph rate \pm & $23.10 \%$ & $16.30 \%$ & $6.8 \%$ Chi-squared: 1.018 & $(-7.4981 \%$ to $16.2177 \%)$ & 0.313 \\
\hline
\end{tabular}

TABLE 2: Statistical analysis of access and accuracy of diagnostic imaging for forearm fractures in pediatric patients aged 3-18 years with stratification by race and healthcare venue

Results stratified by treatment venue (ED vs all other ambulatory primary care venues), revealing a discordance favoring Whites over non-Whites in both imaging rate and the detection of an abnormal radiograph.

* Defined as (total number of patients imaged/total of patients with chief complaint), serves as a proxy to understand the impact of care access.

\pm Defined as (total number of patients diagnosed with a forearm fracture/total number of patients imaged), serves as a proxy to understand impact of unnecessary imaging in pediatric patients.

\section{Discussion}

We found evidence of healthcare disparity in pediatric fracture care in our health system. For traumatic forearm pain in primary care (both in emergency departments - EDs and in ambulatory service lines), nonWhite patients had significantly lower imaging rates than White patients. In addition, non-Whites who were imaged in the ED had lower abnormal radiograph rates than Whites - lower yield imaging in this care venue for non-Whites. The trend is consistent: Whites received more radiography, and that radiography was of higher diagnostic yield.

The difference in imaging rates is perhaps most meaningful in the ED sub-group since, in our study, Whites in EDs were imaged more often and with higher testing yield than non-Whites regardless of trauma. But non-Whites received $4.7 \%$ less imaging for comparable traumatic arm/wrist pain. Moreover, even if nonWhites were imaged, they had a 7.3\% lower abnormal radiograph detection rate. We interpret this as evidence of healthcare disparity [2] - a decrease in both the likelihood of imaging and the likelihood of diagnostic yield among non-White pediatric patients.

In ambulatory sub-group analysis, we found a similar healthcare access disparity - lower imaging rates for non-Whites. However, the difference in abnormal radiograph rate was not statistically significant in the ambulatory sub-group. This difference may be less relevant in the ambulatory setting since most imaging in our study was done in EDs (there was a 4.5 fold greater rate of imaging in the ED compared to ambulatory settings). The interpretation of the ambulatory data should be in the context of care venue bias and workflows (discussed below). Thus, what is most important from the ambulatory perspective is the consistency of racial discordance in imaging rates across care venues. Non-Whites were consistently less likely to be afforded imaging despite equivocal traumatic chief complaints and diagnostic codes, regardless of treatment setting. These imaging rates could be interpreted as a proxy for care access, especially since the inclusion criteria for both racial groups were identical. These findings support the allusion to healthcare access disparity in children with suspected forearm fractures.

Although overall, there were insignificant differences in imaging rates between races, we argue that combining emergency and ambulatory service lines may not be a valid comparison due to differences in workflows in these care venues. For example, suppose a child with traumatic arm pain enters the ED vs. a walk-in medical clinic. In that case, the inherent nature of the venue might play a meaningful role in the treatment approach for the standard of care (i.e., there might be higher rationality in ordering imaging in an emergency setting compared to an ambulatory setting). Support for this concept could be observed by comparing and approximating imaging rates between ambulatory and emergency venues from National Ambulatory Medical Care Survey data [13,14]. Workflow differences in care venues might explain the 60\% higher imaging rate in the ED (Table 2). 
Locally, low yield imaging increases the likelihood of non-Whites encountering financial strain [15]. Historically, and particularly in emergency settings, the literature has supported an increased susceptibility to financial strain on marginalized populations $[3,6,7,16]$. Low yield imaging is medically wasteful; it provides low-value care by increasing the cost burdens like copays, deductibles, and other cost-sharing charges [17]. Although radiography may have small copays or deductibles, such a bill could be a relatively substantial percentage of weekly earnings for low-income families. Moreover, as the literature has described, most children imaged for traumatic arm wrist pain are not diagnosed with fractures, demonstrating the financial futility [9] - a generalizable concept given our population's demographic similarity to US census data [18]. These types of unnecessary cost burdens imposed on vulnerable populations can augment the underlying mechanism of healthcare disparity. Thus, our study identifies a possible feed-forward mechanism that restricts care access and might contribute to disproportionate cost-sharing for non-Whites.

It is unclear why this racial disparity exists. In our study, it may be due to the disproportionately higher use of ED by non-Whites ( $56.9 \%$ vs. $43.6 \%$, Table 1 ). It could also be that fracture rates are higher in Whites, a concept supported by the literature $[19,20]$. Alternatively, perhaps there is an unconscious bias that fuels the known treatment disparity in fracture care [21], or other patient and visit level factors [22]. Regardless, nonWhites received less imaging than Whites in all care venues for painful, traumatic injuries in our study. It was also unclear whether an underlying implicit bias plays a role in the decisions to image non-Whites. Future studies that could detect these concepts would be helpful for understanding structural racism in healthcare.

Identifying these disparities provides an opportunity to discuss improvement strategies for mitigating health inequity. One such strategy is increasing the use of more affordable alternative diagnostic modalities, such as POCUS, in diagnosing forearm fractures. As an established non-inferior diagnostic tool to x-ray, POCUS could be a feasible means to mitigate cost inequity [11]. Especially in rural and underserved care contexts where high racial minority representation exists [4], strategies like POCUS could effectively eliminate cost-sharing since radiologists would not be needed (and thus not billed). Moreover, since rural and underserved areas have lower access to healthcare resources [7], namely radiographic imaging services, implementation of non-radiographic options like POCUS equipment would be a substantially lower cost compared to x-ray, CT, or MRI [17]. Given the high-level evidence specifically supporting the use of POCUS in primary care for fracture diagnosis [23,24], care access for marginalized populations could be increased without the imposition of cost-sharing.

Another strategy could be investing in alternative diagnostic modalities to promote value-based care [25]. Especially in rural practice, ultrasound has lower implementation costs with comparable sensitivity to radiography [26]. Moreover, from a patient perspective, these alternative modalities can reduce harm by decreasing radiation exposure $[27,28]$ and improve the patient experience by reducing psychological stress [29]. The return on investment for practices and health systems could be lower costs, reduction in harm, and mitigation of financial burden for racially marginalized pediatric patients.

\section{Limitations}

First, this study presents evidence of healthcare disparity. We do not know why the imaging rates between White and non-White patients were different. Since traumatic arm/wrist pain was common to both groups, we expected imaging rates to be similar (or at least not different). Our methods lacked the capacity to detect granular data between cohorts (for example, injury mechanism, the severity of pain on presentation, time since injury, social determinants of health, etc.) which would be helpful in ascertaining what the differences between cohorts really means. Similarly, we were unable to determine if there was a correlation between the sub-categories of age and race to know if this influenced the differences in imaging or fracture detection rates; but this would require regression and additional granular data acquisition that was not accessible with our current methodology. Thus, we cannot draw conclusions on care quality and are limited to simply identifying a healthcare disparity.

Second, we could not reliably determine diagnostic accuracy. For example, there was no way of detecting whether fractures were missed or if there is a racial component affecting imaging rates (i.e. if White patients are over-imaged, have weaker bones, or have more exposure to trauma). The only reliable way to conclude diagnostic accuracy would be to review each radiograph in the study, which is beyond the scope of author practice. Thus, the authors reported abnormal radiograph rates in attempts for it to serve as a proxy for medical waste (i.e., the financial impact on marginalized populations).

Lastly, we understand the limitations of our methods in using health system data. In relying on EMR data, there may be uncaptured or inaccurately documented visits. Also, the EMR is not equipped to assess the bias of practitioners ordering the radiography. If implicit bias played a role in racial selection, we did not have a way to measure it. Randomization would undoubtedly provide more substantial evidence to mitigate bias in future studies, which could further evaluate the appropriateness of imaging between races.

\section{Conclusions}

This study presents initial evidence of lower imaging rates in non-White patients for traumatic arm and 
wrist pain compared to White patients. These differences identify a healthcare disparity in pediatric forearm fracture care, both in emergency and ambulatory settings. Implications of these findings call for higher level studies that could investigate the effect of social determinants of health, incorporate more detailed patient data, evaluate the effect of alternative diagnostic modalities, and examine provider bias on facture care equity to understand underlying reasons for observed differences.

\section{Additional Information \\ Disclosures}

Human subjects: All authors have confirmed that this study did not involve human participants or tissue. Animal subjects: All authors have confirmed that this study did not involve animal subjects or tissue. Conflicts of interest: In compliance with the ICMJE uniform disclosure form, all authors declare the following: Payment/services info: All authors have declared that no financial support was received from any organization for the submitted work. Financial relationships: All authors have declared that they have no financial relationships at present or within the previous three years with any organizations that might have an interest in the submitted work. Other relationships: All authors have declared that there are no other relationships or activities that could appear to have influenced the submitted work.

\section{Acknowledgements}

Non-author contributions: Evrhett Davis assisted in the literature review, Steve A Strom was an expert consulted on SlicerDicer data acquisition, Kathryn P Baughman was involved in project conceptualization and manuscript editing. Author contributions: DB and TA conceived the conceptual framework of the project, completed the literature review, and co-authored the introduction. DB orchestrated data acquisition, ran the statistical analysis, and drafted the original manuscript. AW was the principal investigator. AW and TT were essential contributors in data presentation and project mentorship. All authors were editors of the final manuscript.

\section{References}

1. Dieleman JL, Chen C, Crosby SW, et al.: US health care spending by race and ethnicity, 2002-2016. JAMA. 2021, 326:649-59. 10.1001/jama.2021.9937

2. Kaiser Family Foundation: Disparities in health and health care: 5 key questions and answers . (2021). Accessed: September 5, 2021: https://www.kff.org/racial-equity-and-health-policy/issue-brief/disparitiesin-health-and-health-care-5-key-question-....

3. Ortiz SE, Segel JE, Tran LM: Health savings plans and disparities in access to care by race and ethnicity. Am J Prev Med. 2021, 61:e81-92. 10.1016/j.amepre.2021.02.020

4. Racial and ethnic minorities made up about 22 percent of the rural population in 2018 , compared to 43 percent in urban areas. Accessed September 5. (2021). http://www.ers.usda.gov/data-products/chartgallery/gallery/chart-detail/.

5. Newacheck PW, Stoddard JJ, Hughes DC, Pearl M: Health insurance and access to primary care for children . N Engl J Med. 1998, 338:513-9. 10.1056/NEJM199802193380806

6. National Research Council (US) Panel on Race, Ethnicity, and Health in Later Life: Understanding Racial and Ethnic Differences in Health in Late Life: A Research Agenda. Bulatao RA, Anderson NB (ed): National Academies Press (US), Washington DC; 2004. https://www.ncbi.nlm.nih.gov/books/NBK24693/.

7. Thomas Rice: The impact of cost containment efforts on racial and ethnic disparities in health care: a conceptualization. Unequal Treatment: Confronting Racial and Ethnic Disparities in Health Care. Smedley BD, Stith AY, Nelson AR (ed): National Academies Press (US, Washington, DC; 2003.

8. Merrill CT, Owens PL, Stocks C: Pediatric emergency department visits in community hospitals from selected states, 2005. Healthcare Cost and Utilization Project (HCUP) Statistical Briefs [Internet]. Agency for Healthcare Research and Quality (US), Rockville, Maryland; 2008.

9. Tsou PY, Ma YK, Wang YH, Gillon JT, Rafael J, Deanehan JK: Diagnostic accuracy of ultrasound for upper extremity fractures in children: a systematic review and meta-analysis. Am J Emerg Med. 2021, 44:383-94. 10.1016/j.ajem.2020.04.071

10. Herren C, Sobottke R, Ringe MJ, Visel D, Graf M, Müller D, Siewe J: Ultrasound-guided diagnosis of fractures of the distal forearm in children. Orthop Traumatol Surg Res. 2015, 101:501-5. 10.1016/j.otsr.2015.02.010

11. Tanael M: Use of point-of-care ultrasonography in primary care to redress health inequities . J Am Board Fam Med. 2021, 34:853-5. 10.3122/jabfm.2021.04.200452

12. MedCalc: comparison of proportions calculator . (2022). Accessed: September 4, 2021: https://www.medcalc.org/calc/comparison_of_proportions.php.

13. National Hospital Ambulatory Medical Care Survey: 2018 Emergency Department Summary Tables. U.S. Department of Health and Human Services , Centers for Disease Control and Prevention, Atlanta, Georgia; 2018. https://www.cdc.gov/nchs/data/nhamcs/web_tables/2018-ed-web-tables-508.pdf.

14. National Ambulatory Medical Care Survey: 2018 National Summary Tables. U.S. Department of Health and Human Services , Centers for Disease Control and Prevention, Atlanta, Georgia; 2018. https://www.cdc.gov/nchs/data/ahcd/namcs_summary/2018-namcs-web-tables-508.pdf.

15. WellSpan Health: Create a cost estimate . (2021). Accessed: September 21, 2021: https://www.wellspan.org/patients-visitors/patient-guide/billing-insurance/cost-of-care/create-a-costestimate/.

16. Reed M, Fung V, Price M, et al.: High-deductible health insurance plans: efforts to sharpen a blunt instrument. Health Aff (Millwood). 2009, 28:1145-54. 10.1377/hlthaff.28.4.1145

17. Sistrom CL, McKay NL: Costs, charges, and revenues for hospital diagnostic imaging procedures: differences 
by modality and hospital characteristics. J Am Coll Radiol. 2005, 2:511-9. 10.1016/j.jacr.2004.09.013

18. U.S. Census Bureau: QuickFacts Pennsylvania. (2021). Accessed: September 13, 2021 :

https://www.census.gov/quickfacts/PA.

19. Liu LH, Chandra M, Gonzalez JR, Lo JC: Racial and ethnic differences in hip fracture outcomes in men . Am J Manag Care. 2017, 23:560-4.

20. Leslie WD: Clinical review: ethnic differences in bone mass--clinical implications . J Clin Endocrinol Metab. 2012, 97:4329-40. 10.1210/jc.2012-2863

21. Slover J, Gibson J, Tosteson T, Smith B, Koval K: Racial and economic disparity and the treatment of pediatric fractures. J Pediatr Orthop. 2005, 25:717-21. 10.1097/01.bpo.0000184835.79345.0e

22. Wong HJ, Sistrom CL, Benzer TI, et al.: Use of imaging in the emergency department: physicians have limited effect on variation. Radiology. 2013, 268:779-89. 10.1148/radiol.13130972

23. Sorensen B, Hunskaar S: Point-of-care ultrasound in primary care: a systematic review of generalist performed point-of-care ultrasound in unselected populations. Ultrasound J. 2019, 11:31. 10.1186/s13089019-0145-4

24. Andersen CA, Brodersen J, Rudbæk TR, Jensen MB: Patients' experiences of the use of point-of-care ultrasound in general practice - a cross-sectional study. BMC Fam Pract. 2021, 22:116. 10.1186/s12875-02101459-z

25. Shen-Wagner J: Family medicine billing for point-of-care ultrasound (POCUS). J Am Board Fam Med. 2021, 34:856-8. 10.3122/jabfm.2021.04.210187

26. Moritz JD, Berthold LD, Soenksen SF, Alzen GF: Ultrasound in diagnosis of fractures in children: unnecessary harassment or useful addition to X-ray?. Ultraschall Med. 2008, 29:267-74. 10.1055/s-20081027329

27. Cross TM, Smart RC, Thomson JE: Exposure to diagnostic ionizing radiation in sports medicine: assessing and monitoring the risk. Clin J Sport Med. 2003, 13:164-70. 10.1097/00042752-200305000-00007

28. Harvard Health Publishing: Radiation risk from medical imaging . (2010). Accessed: September 5, 2021: https://www.health.harvard.edu/cancer/radiation-risk-from-medical-imaging.

29. Bradford R: Short communication: the importance of psychosocial factors in understanding child distress during routine x-ray procedures. J Child Psychol Psychiatry. 1990, 31:973-82. 10.1111/j.14697610.1990.tb00838.x 